\title{
Computational Analysis of Knee Joint Stability Following Total Knee Arthroplasty Using Bode Margins
}

Marzieh M Ardestani ${ }^{1}$, ZhenXian Chen ${ }^{2}$, Hessam Noori ${ }^{3}$, Mehran Moazen ${ }^{4}$, Zhongmin

$$
\operatorname{Jin}^{2,5,6}
$$

${ }^{1}$ Department of Physical Medicine and Rehabilitation, School of Medicine, Indiana University, IN, USA

${ }^{2}$ School of Mechanical Engineering, Southwest Jiaotong University, Chengdu, China ${ }^{3}$ School of Mechanical and Energy Engineering, Purdue University, Purdue, IN

${ }^{4}$ Department of Mechanical Engineering, University College London, Torrington Place, London WC1E 7JE, UK

${ }^{5}$ School of Mechanical Engineering, Xian Jiaotong University, Xian, China

${ }^{6}$ School of Mechanical Engineering, University of Leeds, Leeds, LS2 9JT, UK

*Address for corresponding author:

Marzieh M. Ardestani

Assistant Research Professor

Department of Physical medicine and Rehabilitation

School of Medicine

Indiana University-Purdue University

4141 Shore Dr

Indianapolis, IN, 46254

Email: mamost@iu.edu 


\section{Abstract}

The overall objective of this study was to introduce knee joint power as a potential measure to investigate knee joint stability following total knee arthroplasty (TKA). Specific aims were to investigate whether weakened knee joint stabilizers cause abnormal kinematics and how it influences the knee joint kinetic (i.e., power) in response to perturbation.

Patient-specific musculoskeletal models were simulated with experimental gait data from six TKA patients (baseline models). Muscle strength and ligament force parameter were reduced by up to $30 \%$ to simulate weak knee joint stabilizers (weak models). Two different muscle recruitment criteria were tested to examine whether altered muscle recruitment pattern can mask the influence of weakened stabilizers on the knee joint kinematics and kinetics. Level-walking knee joint kinematics and kinetics were calculated though force-dependent kinematic and inverse dynamic analyses. Bode analysis was then recruited to estimate the knee joint power in response to a simulated perturbation.

Weak models resulted in larger anterior-posterior (A-P) displacement and internal-external (I-E) rotation compared to baseline (I-E: $18.4 \pm 8.5$ vs. $11.6 \pm 5.7$ (deg), A-P: $9.7 \pm 5.6$ vs. $5.5 \pm 4.1$ (mm)). Changes in muscle recruitment criterion however altered the results such that A-P and I-E were not notably different from baseline models. In response to the simulated perturbation, weak models versus baseline models generated a delayed power response with unbounded magnitudes. Perturbed power behavior of the knee remained unaltered regardless of the muscle recruitment criteria.

In conclusion, impairment at the knee joint stabilizers may or may not lead to excessive joint motions but it notably affects the knee joint power in response to a perturbation. Whether perturbed knee joint power is associated with the patient-reported outcome requires further investigation.

Keywords: Total knee arthroplasty, instability, knee kinematics, gait, Bode analysis 


\section{Introduction}

Instability of total knee arthroplasty (TKA) causes 20-30\% of the implanted knees to be revised annually (Parratte and Pagnano, 2008; Rodriguez-Merchan, 2011). Accurate diagnosis is therefore crucial to plan the revision surgery (Kanamiya et al., 2002; Matsuda and Ito, 2015). However, the diagnosis can be challenging; e.g., 8-20\% of TKA patients complain about persistent instability in the absence of any immediate symptoms (Azzam et al., 2011; Sharkey et al., 2014; Song et al., 2014). Persistent, yet asymptomatic, knee instability is often attributed to insufficiency of the knee joint stabilizers; i.e., lax ligaments and/or weak muscles. Impaired knee joint stabilizers can cause abnormal, often unbounded kinematics and/or kinetics in response to a bounded perturbation (Bergmark, 1989).

Hypermobility, i.e., excessive anterior-posterior (A-P) displacement (Fantozzi et al., 2006; Stoddard et al., 2013) and/or internal-external (I-E) rotation (Wautier and Thienpont, 2017; Zaffagnini et al., 2014) is a familiar manifest of the unbounded kinematic response of an unstable knee. Yet, whether the diagnosis of instability should be excluded when hypermobility is not observed during clinical assessments is a matter of debate (Martín-Hernández et al., 2014; Nakahara et al., 2015).

Classic clinical assessments of hypermobility such as anterior drawer test, Lachman evaluation and pivot shift test apply subjective perturbations (Athwal et al., 2014). Recent studies recommended alternative evaluations of the knee joint kinematics under a more dynamic condition such as level walking or stair navigation to evoke hypermobility (Denney et al., 2014; Joglekar et al., 2012; Soeno et al., 2018). Nonetheless, human neuro-musculoskeletal system is capable of adopting a compensatory muscle recruitment strategy (i.e., redundancy) such that kinematic and kinetic behavior, especially in a low-demanding task such as level-walking, remains unaltered 
(Bonnefoy-Mazure et al., 2017; Liebensteiner et al., 2008; Soeno et al., 2018). Our recent study showed that TKA patients with sub-optimal knee function may still demonstrate asymptomatic knee kinematics, owing to compensatory muscle recruitment patterns (Ardestani et al., 2017).

Abnormal kinetic behavior in response to perturbation, e.g., unbounded joint power can be another manifest of instability (Levin et al., 2015; Vera-Garcia et al., 2007). This concept however is overlooked in TKA studies. One explanation can be that any perturbation may damage the prosthetic knee and thus may not be applied due to ethical considerations. Besides, the perturbation, required to evoke the unbounded behavior, might be patient-specific. Bode analysis is a well-documented technique in control engineering (Ogata and Yang, 2002) capable of simulating a perturbation and then qualitatively estimating the perturbed behavior of a system to determine its stability margins, often referred as "Bode margins". Bode analysis estimates the perturbed behavior of a system based on its unperturbed dynamic(Dorf and Bishop, 2011), and thus relaxes the necessity of applying an actual perturbation to the system (i.e., the knee joint). Additionally, Bode analysis often simplifies a complex system to a linear function with few inputs and outputs. For instance, the knee joint can be modeled as a linear function with the knee joint kinematics and kinetics as inputs and the knee joint power as output facilitating the estimation of the perturbed knee joint power. Bode analysis was recently used to estimate the perturbed kinematic behavior of unstable knees following anterior-cruciate ligament injury (Morgan et al., 2016).

The overall objective of this study was to investigate the applicability of Bode analysis to estimate the perturbed knee joint power in TKA patients. We aimed to investigate whether weakened knee joint stabilizers cause abnormal kinematics during walking (hypermobility) and 
how it can influence the knee joint kinetics (power) in response to larger perturbations beyond level-walking.

\section{Materials and Methods}

Six TKA patients were obtained from a published repository (Section 2.1). Our previously published musculoskeletal (MSK) model of a typical TKA patient was scaled to each patient (Section 2.2). Two separate versions of MSK models were developed: (i) baseline (BSL) models with intact joint stabilizers (muscles and ligaments) and (ii) weakened (WEAK) models for which the knee muscle strength and ligament force parameter were reduced. For each patient, BSL and WEAK models were simulated with the averaged level-walking gait profile (ground reaction force and marker trajectories) of that patient. Inverse dynamic and Force-dependent kinematic (FDK) analyses were conducted to calculate knee joint kinetics and the secondary knee joint kinematics (A-P displacement and I-E rotation) respectively. This was performed to investigate whether weakened knee joint stabilizers immediately lead to abnormal pattern in the secondary knee joint kinematics (hypermobility). The knee joint kinematics and kinetics from BSL and WEAK models were then imported to Bode analysis to estimate the knee joint power in response to the simulated perturbation (Section 2.3). This was performed to investigate whether weakened joint stabilizers can impact stability margins of the knee joint in response to the perturbation. Figure 1 demonstrates the workflow of the present study. 


\subsection{Experimental Gait Data}

Gait data including ground reaction forces (GRF) and marker trajectories from six TKA patients (5 M/ 1F, Height: $170.8 \pm 5.2 \mathrm{~cm}$; Weight: $69.7 \pm 4.4 \mathrm{~kg}$ ) were obtained from a published repository (https://simtk.org/home/kneeloads, accessed Sept 2015). TKA patients were implanted with cruciate-retaining sensor-based knee prostheses which measures in vivo knee forces. GRFs were recorded at a frequency of $1000 \mathrm{~Hz}$ (Force plate, AMTI Corp., Watertown, MA, USA) and marker trajectory data were recorded at a frequency of $200 \mathrm{~Hz}$ (10-camera motion capture system, Motion Analysis Corp., Santa Rosa, CA, USA) using a modified Cleveland Clinic marker set with extra markers on the feet and trunk. For a complete description of this database see (Fregly et al., 2012; Kinney et al., 2013).

\subsection{Musculoskeletal Model}

We previously modified a 3D musculoskeletal model, i.e., Twente Lower Extremity Model (TLEM) model (Horsman, 2007), from AnyBody software repository (version 6.0; AnyBody Technology, Aalborg, Denmark) to represent a TKA patient(Chen et al., 2016a; Chen et al., 2015; Chen et al., 2014; Chen et al., 2016b). TLEM, with 160 muscle-tendon actuators, spherical hip and revolute knee and ankle joints were modified as follows: The generic geometry of the knee (femoral and tibial components) was replaced with the geometry of the knee implant (Figure 2). Two deformable contact models were defined between the tibial insert and femoral component bearing surfaces and between the patellar button and the femoral component. A friction coefficient of 0.04 was considered between the two components (Hashemi et al., 2000). Details of these contact models are discussed in the Appendix. This model solves the equilibrium equations in three dimensions(Damsgaard et al., 2006). The model showed acceptable accuracy in predicting muscle activations and the knee joint contact forces when compared versus in-vivo measurements 
(Chen et al., 2014; Peng et al., 2018). This model was also used for a series of parametric and probabilistic studies (Ardestani and Moazen, 2016; Chen et al., 2015). Here, this model was used to simulate muscle weakness (strength decline) and ligament laxity (decline in ligament force parameter) and calculate the resultant knee joint kinematics and kinetics.

The MSK model was scaled to each patient as follows: model was scaled to each patient's weight and height using Length-Mass-Fat scaling law(Lund et al., 2015). Body segment lengths and the relative positions of joints were determined such that the model's markers closely tracked the experimental marker trajectories. Maximum isometric voluntary contractions of muscles $\left(F_{0}\right)$ were also scaled using Height-Squared law(Rasmussen et al., 2005). Muscle attachment and geometries were scaled based on linear geometry scaling law (Worsley et al., 2011). Muscle strength was represented using a bilinear model (Lloyd and Besier, 2003):

Strength $=F_{0}\left(2 \frac{L_{m}}{L_{f}}-1\right)\left(1-\frac{L_{m}{ }^{\prime}}{V_{0}}\right)$

Where $F_{0}$ is the strength of the muscle at neutral fiber length $\left(L_{f}\right)$ and contraction velocity $\left(L^{\prime}{ }_{m}\right)$ equals to zero. $L_{m}$ is the current length of the contractile element and $V_{0}$ is the contraction velocity at maximum voluntary contraction. $F_{0}$ is related to muscle isometric strength and has been estimated from cadaveric studies (Horsman, 2007) Muscle weakness was simulated by reducing the strength parameter, $F_{0}$ for the following muscles : semimembranosus, semitendinosus, biceps femoris, rectus femoris, vastus, tibialis anterior, medial gastrocnemius and soleus.

Ligaments, including posterior cruciate ligament (PCL), medial collateral ligament (MCL), lateral collateral ligament (LCL), posteromedial capsule (PMC), medial PF ligament (MPFL), and lateral PF ligament (LPFL), were modeled as non-linear spring elements with the piecewise force- 
displacement relationship (Blankevoort, 2001) (Note: anterior cruciate ligament (ACL) was not modeled considering the surgical removal of this ligament):

$$
f=\left\{\begin{array}{lr}
S_{0} \times\left(\frac{\varepsilon^{2}}{4 \varepsilon_{l}}\right) & 0<\varepsilon<2 \varepsilon_{l} \\
S_{0} \times\left(\varepsilon-\varepsilon_{l}\right) & \varepsilon>2 \varepsilon_{l} \\
0 & \varepsilon<0
\end{array}\right.
$$

$$
\varepsilon=\frac{L-L_{0}}{L_{0}} \quad, \quad L_{0}=\frac{L_{r}}{\varepsilon_{r}+1}
$$

where $f$ is the ligament force and $S_{0}$ is the ligament force parameter, expressed in newton, $\varepsilon_{l}$ is a constant non-linear strain parameter of $0.03, \varepsilon$ is the strain in the ligaments, $L$ is the ligament length, and $L_{0}$ is the zero-load length of the ligament (determined from the ligament's initial length $L_{r}$ and the reference strain $\varepsilon_{r}$ ). Ligament laxity was simulated by reducing ligament force parameter, $S_{0}$, in equation (2).

From each patient-specific model, 400 versions were generated including (i) $100 \mathrm{BSL}$ models, for which muscle strength and ligament force parameter were chosen from a normal distribution of the nominal values for that subject $\pm 5 \%$ (Amiri and Wilson, 2012), (ii) 100 WEAK models where $F_{0}$ was chosen from a normal distribution of nominal strength reduced by $30 \%$ (Silva et al., 2003). Note our previous study showed reduction beyond $40 \%$ can alter the normal gait pattern (Ardestani and Moazen, 2016); (iii) 100 WEAK models with lax ligaments where $S_{0}$ was chosen from normal distribution of nominal values reduced by $30 \%$. This is consistent with previous literature reporting up to $30 \%$ of variation in ligament stiffness amongst TKA subjects with unstable knees (Reinders et al., 2014) which may in turn lead to 2 standard deviation in secondary knee joint kinematics from the average (Kang et al., 2017). Note previous studies showed that reduction beyond 50\% can alter the joint load (Li et al., 2002; Orozco et al., 2018), 
(vi) 100 WEAK models with both weak muscles and lax ligaments. For BSL models, $F_{0}$ and $S_{0}$ values were consistent with reported values for stable knees (Anderson and Pandy, 1999; Lin et al., 2010) - Table 1). For each patient, both BSL and WEAK models were simulated with the average marker trajectory and GRF profile of the same patient. Inverse dynamic analysis was conducted to calculate the joint moments and muscle forces from GRF and primary joint kinematics. Furthermore, force-dependent kinematic (FDK) analysis was conducted to calculate the secondary knee joint kinematics and internal joint contact forces. For FDK analysis, please see (Andersen et al., 2011). In brief, FDK analysis was conducted by introducing an additional kinematic driver to a standard inverse dynamic analysis. The kinematic driver was the function of joint coordination and time. This was added to represent the fact that in a nonconforming joint such as knee, internal forces influence joint secondary kinematics. The time-derivate of this kinematic drive was assumed to be zero so that the equilibrium equations become quasi-static. The underlying assumption of FDK analysis was that the secondary knee motions were not influenced by the global model dynamics and therefore, can be solved assuming quasi-static equilibrium between ligament, muscle, contact forces, and external loads.

Two different muscle recruitment criteria were implemented: (1) the conventional Min-Max optimization which activates the muscles such that minimizes the maximum muscle activation(Marra et al., 2015); (2) a recently proposed synergy optimization which activates muscles to minimize synergy activations (instead of muscle activation) where synergy is defined as phase-specific groups of agonist muscles (Aoi and Funato, 2016; Sartori et al., 2013). Presented by new evidence and confirmed by our recent study (Ardestani et al., 2017), synergistic recruitment of muscles enables the MSK system to accommodate certain levels of muscle weakness and maintain asymptomatic joint kinematics. Therefore, the latter optimization was 
implemented to investigate whether changes in muscle recruitment pattern can mask muscle weakness and prevent the manifest of abnormal knee kinematics during level-walking.

\subsection{Bode Analysis}

For the purpose of Bode analysis, knee joint was represented as a linear model with sagittal knee angular velocity and sagittal knee joint moment as inputs and knee joint power as output. Two-thirds of the BSL simulations were used to construct this model and the remaining one-third of BSL simulations were used to validate it. At least $85 \%$ accuracy $\left(\mathrm{R}^{2} \geq 0.85\right)$ between the knee joint power (output) calculated from the linear model and MSK model was required to deem the linear model of knee as acceptable. This process was conducted using System Identification Toolbox (MATLAB software. 2014b, Chicago, USA).

Once the knee joint was formulated, the inputs (motion and moment) were perturbed and the model was recruited to predict the knee joint power (output) in response to the perturbed inputs. Perturbation was modeled as a sudden change in the knee joint motion and/or moment. The ratio of the resultant knee joint power in response to the perturbed knee flexion angle and/or moment was calculated and referred as "amplitude response". The temporal delay between when the perturbation occurred in the input and when the knee joint responded, was also calculated and referred as "phase response".

Amplitude and phase responses of the knee joint was calculated across a range of different perturbations (frequencies) and was considered as "frequency response" of the knee joint. Bode plot displays the amplitude response vs. frequency and the phase response vs. frequency of the knee joint (Figure 3). Stability margins were obtained from the Bode plot, namely (1) gain margin and (2) phase margin (Figure 3): 
where $G$ is the amplitude response of the knee joint in decibel $(\mathrm{dB})$ at a perturbation for which the phase response of knee joint equals to -180 deg indicating that knee generated the power with half a cycle delay in response to perturbation.

where $P$ is the phase response of the knee joint (in degrees) at a perturbation for which its amplitude response equals to $0 \mathrm{~dB}$ indicating that the output and input amplitudes of the knee joint are equal. As suggested in control engineering, negative amplitude margin and/or negative phase margin indicate an unstable system (Dorf and Bishop, 2011; Ogata and Yang, 2002).

\section{Results}

\subsection{Secondary Knee Joint Motions in BSL vs. WEAK models}

FDK analyses of BSL models, with nominal muscle strength and ligament force parameter, led to an average I-E rotation of $11.6 \pm 5.7$ (deg) and A-P displacement of 5.5 $\pm 4.1(\mathrm{~mm})$. FDK analyses of models with weak muscles (Figure 4) led to slightly higher I-E rotation $(15.7 \pm 8.4$ (deg)) and A-P displacement $(8.3 \pm 5.8(\mathrm{~mm}))$. Models with lax ligaments also led to larger knee joint motions (I-E: $15.3 \pm 5.4(\mathrm{deg})$, A-P:6.7 $\pm 5.7(\mathrm{~mm})$ ). Models with simultaneous muscle weakness and ligament laxity resulted in even larger ranges of knee motions (I-E: 18.4 $\pm 8.5(\mathrm{deg})$, A-P:9.7 $\pm 5.6(\mathrm{~mm}))$. Switching the cost function from Min-Max to synergy optimization changed the muscle recruitment patterns and thus the secondary motions of the knee joint such that results were not notably different from BSL MSK models (Figure 4 and Table 2).

Note, WEAK models compared to BSL models led to large standard deviations in the knee joint motion. Subset analyses of models showed that only models with the reduction in muscle 
strength by $30 \%$, or reduction in ligament force parameter by $18 \%$ caused recognizable deviation (i.e., more than one std) from BSL models. WEAK models with simultaneous reduction in muscle strength (>24\%) and in ligaments force parameter $(>15 \%)$ led to even larger kinematic deviation from BSL models. In this subset of MSK models, switching the cost function from Min-Max to synergy optimization decreased the kinematic deviations but the kinematics remained marginally significant from BSL models (Table 2).

\subsection{Bode Margins in BSL vs. WEAK Models}

Bode analysis of all BSL models led to positive stability margins (i.e., positive amplitude margin and positive phase margin) with amplitude margins $(\mathrm{G})$ ranging from $5.8(\mathrm{~dB})$ to $22.5(\mathrm{~dB})$ and phase margins (P) ranging from 35.7 (deg) to 136.8(deg) indicating a promptly-generated power with bounded amplitude in response to perturbation (Figure 5a). In contrast, $85 \%$ of WEAK models with reduced muscle strength, ligament force parameter or both led to negative stability margins in Bode analysis. More directly, $88 \%$ of WEAK models with reduced muscle strength led to negative amplitude margins $(\mathrm{G}=-15.8 \pm 13.5(\mathrm{~dB}))$ indicating an unbounded power response to perturbation (Figure 5b). On the other hand, $82 \%$ of WEAK models with reduced ligament force parameter led to negative phase margins $(\mathrm{P}=-65.3 \pm 23.7(\mathrm{deg}))$ indicating a delayed power behavior in response to perturbation (Figure 5c). Note $12 \%$ of models with reduced muscle strength and $18 \%$ of those with reduced ligament force parameter still led to positive, albeit small, stability margins $(\mathrm{G}=2.3 \pm 2.7(\mathrm{~dB}), \mathrm{P}=11.4 \pm 6.9(\mathrm{deg}))$. A closer investigating of these models revealed that manipulated parameters were close to nominal thresholds (strength reduction less than $10 \%$ and ligament force parameter reduction less than $8 \%$ ). Switching the cost function from Min-Max to synergy optimization decreased the prevalence of negative stability margins from $88 \%$ to $72 \%$ (and from $82 \%$ to $78 \%$ ) in models with reduced strength (and models with reduced 
ligament force parameter). Yet, the prevalence of negative Bode margins in WEAK models remained notable.

Bode analyses of WEAK models with simultaneous reductions of muscle strength and ligament force parameter led to negative stability margins indicating a delayed $(\mathrm{P}=-97.8 \pm 32(\mathrm{deg}))$ and unbounded $(\mathrm{G}=-23.8 \pm 14.6(\mathrm{~dB}))$ power response to perturbation (Figure $5 \mathrm{~d})$. In these models, Bode estimations of the knee power were consistent regardless of the muscle recruitment function; i.e., changing the muscle recruitment function from the Min-Max optimization to the synergy optimization slightly changed the magnitude and the delay in joint power, but WEAK models still showed a delayed power behavior with unbounded magnitude in response to perturbation (see Figure 5). Samples of Bode plots are presented in the Appendix.

\section{Discussion}

This study recruited Bode analysis to qualitatively estimate the knee joint power in response to a simulated perturbation. We aimed to investigate whether weakness (up to 30\%) in the ligament and muscles immediately cause abnormal knee kinematics during level walking (hypermobility) and whether impair the kinetic behavior (power) in response to the larger perturbations. Two different muscle recruitment criteria were also tested to examine whether altered muscle recruitment pattern can mask the influence of weak stabilizers on the knee joint kinematics and kinetics. Results showed that depending on the muscle recruitment pattern, weak knee joint stabilizers may or may not cause excessive joint motions, but it notably affects the knee joint power in response to the perturbation.

Computational analyses of the knee joint stability advance our understanding of the isolated and combined roles of knee joint kinematics and kinetics, muscle co-activation or 
anatomical variables on the knee joint stability (Sharifi et al., 2018). Significant reduction in ligament stiffness and/or muscle activation has been shown to change the knee joint kinematics and causes hypermobility (Sharifi et al., 2017). Some patients however may demonstrate asymptomatic knee kinematics during walking while presenting with persistent complaints of knee instability and dysfunction (Ardestani et al., 2017). The present study therefore focused on only small levels of muscle weakness and ligament laxity. Considering the redundancy of human MSK system, we aimed to demonstrate that impairment at the knee stabilizers may be compensated through altered muscle recruitment such that it may not immediately translate into abnormal kinematics. Yet, it may impair the ability of the knee joint to respond to a perturbation beyond level walking. Morgan et al used Bode analysis to discuss the abnormal knee kinematic in response to perturbation and the present study focused on perturbed kinetic behavior.

The knee joint power was studied as the kinetic behavior of interest. The knee joint power integrates the role of both kinematics (dictated by passive constraints such as ligaments) and kinetics (dictated by active constraints i.e., muscles) and hence is expected to be more informative to manifest knee joint complications. Besides, the knee joint power is calculated as the dot product of the joint moment and the angular velocity (the derivation of sagittal knee motion). Sagittal knee joint motion is the dominant movement of the knee joint and mid-flexion instability is the most popular type of instability. Moreover, daily-life activities often induce perturbation which can be modeled as sudden changes in the movement (e.g., rapid turn) and/or sudden changes in the ground reaction forces (e.g., uneven ground, slippery surfaces) influencing the knee joint moment.

This study has several limitations. First the computational approach was based on a small patient population. Nonetheless, random selection of the key variables including muscle strength and ligament force parameter created a large probabilistic data base (400 simulations per each 
subject). Future experimental investigations with a larger TKA population is required to confirm present findings.

Second, computational modeling including MSK modeling and Bode analysis bring their inherent limitations. The origin and insertion sites of the muscles and the ligaments were based on TLEM model and may not exactly represent individual patients. Although MSK models were scaled to each patient, other properties such as muscle activation, muscle cross-sectional area and ligament geometries were not adjusted to individual age and their unique anatomy. Moreover, muscle weakness was solely simulated by decreasing the strength. Reduction in muscle crosssectional area and muscle fiber excitability are other etiologies that may also lead to muscle weakness. Also, muscle weakness was only simulated in eight muscles. It should be noted that other muscles, even those that are not directly connected to the knee, may also influence the knee joint loads and its motions. Muscle-tendon units were simplified using a bilinear model (equation 1). This model consists of a contractile element and a serial-elastic element. Unlike a hill-type model, the bilinear model does not have a parallel elasticity element to account for passive muscle force. Instead, this model uses larger isometric force parameters compared to hill-type model To account for passive muscle force. This built-in passive force however cannot be switch off and may leads to over-estimated muscle strength and thus muscle forces. This model was chosen as it is computationally efficient for probabilistic studies.

Furthermore, the knee joint stabilizers (ligaments and muscles) were weakened according to pre-determined thresholds and from a normal probability distribution. These assumptions may not necessarily represent an "unstable knee" but rather an increased likelihood of instability. Note, both BSL and WEAK models were simulated using the same marker trajectory data. Therefore knee F-E rotation and the overall kinematic pattern of walking calculated based on marker 
trajectories were assumed to be the same for both models (Thompson et al., 2013; van der Krogt et al., 2012). In real world however, changes in the joint internal structure can influence all joint kinematics in all planes. Furthermore, Bode analysis simplifies the knee by a linear approximation to computationally simulate perturbation and to qualitatively estimate system's behavior in frequency domain. Bode analysis is not a quantitative approach and interpretation of its result in time domain should be conducted with caution.

Finally, further investigations are required to provide a one-by-one comparison between the knee joint power, knee joint kinematic and Bode margins in presence of a real perturbation. Questions such as whether Bode margins are negative (or respectively positive) for patients with confirmed knee instability (or for uninjured knee joints) remains unanswered.

In summary this study explored the application of Bode analysis to estimate the knee joint power in response to a simulated perturbation. Impairment at the knee joint stabilizers can potentially impair the knee joint power in response to the perturbation regardless of the muscle recruitment pattern.

\section{Conflict of interest}

The authors have no financial or non-financial competing interests relevant to this manuscript.

\section{Acknowledgements}

Authors would like to include a special note of thanks for Dr Aaron G. Rosenberg and Dr Markus

A. Wimmer for contributing their valuable time and thoughts to discuss this topic. 
Amiri, S., Wilson, D.R., 2012. A computational modeling approach for investigating soft tissue balancing in bicruciate retaining knee arthroplasty. Computational and mathematical methods in medicine 2012. Andersen, M.S., Damsgaard, M., Rasmussen, J., Year Force-dependent kinematics: a new analysis method for non-conforming joints. In XIII International Symposium on Computer Simulation in Biomechanics, Leuven, Belgium.

Anderson, F.C., Pandy, M.G., 1999. A dynamic optimization solution for vertical jumping in three dimensions. Computer methods in biomechanics and biomedical engineering 2, 201-231.

Aoi, S., Funato, T.J.N.r., 2016. Neuromusculoskeletal models based on the muscle synergy hypothesis for the investigation of adaptive motor control in locomotion via sensory-motor coordination. 104, 88-95. Ardestani, M.M., Malloy, P., Nam, D., Rosenberg, A.G., Wimmer, M.A., 2017. TKA patients with unsatisfying knee function show changes in neuromotor synergy pattern but not joint biomechanics. Journal of Electromyography and Kinesiology 37, 90-100.

Ardestani, M.M., Moazen, M.J.J.o.b., 2016. How human gait responds to muscle impairment in total knee arthroplasty patients: muscular compensations and articular perturbations. 49, 1620-1633.

Athwal, K.K., Hunt, N.C., Davies, A.J., Deehan, D.J., Amis, A.A., 2014. Clinical biomechanics of instability related to total knee arthroplasty. Clinical Biomechanics 29, 119-128.

Azzam, K., Parvizi, J., Kaufman, D., Purtill, J.J., Sharkey, P.F., Austin, M.S., 2011. Revision of the unstable total knee arthroplasty: outcome predictors. The Journal of arthroplasty 26, 1139-1144.

Bergmark, A., 1989. Stability of the lumbar spine: a study in mechanical engineering. Acta Orthopaedica Scandinavica 60, 1-54.

Blankevoort, L.J.J.o.B., 2001. Articular contact in a three dimensional model of the knee. 34, 859-871. Bonnefoy-Mazure, A., Armand, S., Sagawa, Y., Suvà, D., Miozzari, H., Turcot, K., 2017. Knee kinematic and clinical outcomes evolution before, 3 months, and 1 year after total knee arthroplasty. The Journal of arthroplasty 32, 793-800.

Chen, Z., Jin, Z.J.B., Biotribology, 2016a. Prediction of in-vivo kinematics and contact track of total knee arthroplasty during walking. 2, 86-94.

Chen, Z., Wang, L., Liu, Y., He, J., Lian, Q., Li, D., Jin, Z., 2015. Effect of component mal - rotation on knee loading in total knee arthroplasty using multi - body dynamics modeling under a simulated walking gait. Journal of Orthopaedic Research 33, 1287-1296.

Chen, Z., Zhang, X., Ardestani, M.M., Wang, L., Liu, Y., Lian, Q., He, J., Li, D., Jin, Z., 2014. Prediction of in vivo joint mechanics of an artificial knee implant using rigid multi-body dynamics with elastic contacts. Proceedings of the Institution of Mechanical Engineers, Part H: Journal of Engineering in Medicine 228, 564-575.

Chen, Z., Zhang, Z., Wang, L., Li, D., Zhang, Y., Jin, Z.J.M.e., physics, 2016b. Evaluation of a subject-specific musculoskeletal modelling framework for load prediction in total knee arthroplasty. 38, 708-716.

Damsgaard, M., Rasmussen, J., Christensen, S.T., Surma, E., De Zee, M.J.S.M.P., Theory, 2006. Analysis of musculoskeletal systems in the AnyBody Modeling System. 14, 1100-1111.

Denney, L.M., Ferris, L.A., Dai, H., Maletsky, L.P., 2014. Analysis of a rotary task following total knee arthroplasty: Stair descent with a cross-over turn. Proceedings of the Institution of Mechanical Engineers, Part H: Journal of Engineering in Medicine 228, 429-438.

Dorf, R.C., Bishop, R.H., 2011. Modern control systems. Pearson.

Fantozzi, S., Catani, F., Ensini, A., Leardini, A., Giannini, S., 2006. Femoral rollback of cruciate - retaining and posterior - stabilized total knee replacements: in vivo fluoroscopic analysis during activities of daily living. Journal of orthopaedic research 24, 2222-2229. 
Fregly, B.J., Besier, T.F., Lloyd, D.G., Delp, S.L., Banks, S.A., Pandy, M.G., D'lima, D.D., 2012. Grand challenge competition to predict in vivo knee loads. Journal of Orthopaedic Research 30, 503-513. Hashemi, A., Shirazi-Adl, A.J.C.m.i.b., engineering, b., 2000. Finite element analysis of tibial implantseffect of fixation design and friction model. 3, 183-201.

Horsman, K., 2007. The Twente lower extremity model. Consistent dynamic simulation of the human locomotor apparatus.

Joglekar, S., Gioe, T.J., Yoon, P., Schwartz, M.H., 2012. Gait analysis comparison of cruciate retaining and substituting TKA following PCL sacrifice. The Knee 19, 279-285.

Kanamiya, T., Whiteside, L.A., Nakamura, T., Mihalko, W.M., Steiger, J., Naito, M., 2002. Effect of Selective Lateral Ligament Release on Stability in Knee Arthroplasty. Clinical orthopaedics and related research 404, 24-31.

Kang, K., Koh, Y., Jung, M., Nam, J., Son, J., Lee, Y., Kim, S., Kim, S.J.B., research, j., 2017. The effects of posterior cruciate ligament deficiency on posterolateral corner structures under gait-and squat-loading conditions: A computational knee model. 6, 31-42.

Kinney, A.L., Besier, T.F., D'Lima, D.D., Fregly, B.J., 2013. Update on grand challenge competition to predict in vivo knee loads. Journal of biomechanical engineering 135, 021012.

Levin, O., Vanwanseele, B., Thijsen, J.R., Helsen, W.F., Staes, F.F., Duysens, J., 2015. Proactive and reactive neuromuscular control in subjects with chronic ankle instability: evidence from a pilot study on landing. Gait \& posture 41, 106-111.

Li, G., Suggs, J., Gill, T.J.A.o.b.e., 2002. The effect of anterior cruciate ligament injury on knee joint function under a simulated muscle load: a three-dimensional computational simulation. 30, 713-720.

Liebensteiner, M., Herten, A., Gstoettner, M., Thaler, M., Krismer, M., Bach, C., 2008. Correlation between objective gait parameters and subjective score measurements before and after total knee arthroplasty. The Knee 15, 461-466.

Lin, Y.-C., Walter, J.P., Banks, S.A., Pandy, M.G., Fregly, B.J., 2010. Simultaneous prediction of muscle and contact forces in the knee during gait. Journal of biomechanics 43, 945-952.

Lloyd, D.G., Besier, T.F.J.J.o.b., 2003. An EMG-driven musculoskeletal model to estimate muscle forces and knee joint moments in vivo. 36, 765-776.

Lund, M.E., Andersen, M.S., de Zee, M., Rasmussen, J.J.I.B., 2015. Scaling of musculoskeletal models from static and dynamic trials. 2, 1-11.

Marra, M.A., Vanheule, V., Fluit, R., Koopman, B.H., Rasmussen, J., Verdonschot, N., Andersen, M.S.J.J.o.b.e., 2015. A subject-specific musculoskeletal modeling framework to predict in vivo mechanics of total knee arthroplasty. 137, 020904.

Martín-Hernández, C., Revenga-Giertych, C., Hernández-Vaquero, D., Albareda-Albareda, J., QueirugaDios, J., García-Aguilera, D., Ranera-García, M., 2014. Does the medial-lateral stability of total knee replacements have an effect on short-term clinical outcomes? One-year results of a multicentre study with computer assisted surgery. Revista Española de Cirugía Ortopédica y Traumatología (English Edition) 58, 101-107.

Matsuda, S., Ito, H., 2015. Ligament balancing in total knee arthroplasty-Medial stabilizing technique. Asia-Pacific Journal of Sports Medicine, Arthroscopy, Rehabilitation and Technology 2, 108-113.

Morgan, K.D., Zheng, Y., Bush, H., Noehren, B., 2016. Nyquist and Bode stability criteria to assess changes in dynamic knee stability in healthy and anterior cruciate ligament reconstructed individuals during walking. Journal of biomechanics 49, 1686-1691.

Nakahara, H., Okazaki, K., Hamai, S., Okamoto, S., Kuwashima, U., Higaki, H., Iwamoto, Y., 2015. Does knee stability in the coronal plane in extension affect function and outcome after total knee arthroplasty? Knee Surgery, Sports Traumatology, Arthroscopy 23, 1693-1698.

Ogata, K., Yang, Y., 2002. Modern control engineering. Prentice hall India. 
Orozco, G.A., Tanska, P., Mononen, M.E., Halonen, K.S., Korhonen, R.K.J.S.r., 2018. The effect of constitutive representations and structural constituents of ligaments on knee joint mechanics. 8, 2323. Parratte, S., Pagnano, M.W., 2008. Instability after total knee arthroplasty. JBJS 90, 184-194. Peng, Y., Zhang, Z., Gao, Y., Chen, Z., Xin, H., Zhang, Q., Fan, X., Jin, Z.J.M.e., physics, 2018. Concurrent prediction of ground reaction forces and moments and tibiofemoral contact forces during walking using musculoskeletal modelling. 52, 31-40.

Rasmussen, J., de Zee, M., Damsgaard, M., Christensen, S.T., Marek, C., Siebertz, K., Year A general method for scaling musculo-skeletal models. In 2005 International Symposium on Computer Simulation in Biomechanics, Cleveland, $\mathrm{OH}$.

Reinders, J., Sonntag, R., Kretzer, J.P.J.B.r.i., 2014. Wear behavior of an unstable knee: stabilization via implant design? 2014.

Rodriguez-Merchan, E.C., 2011. Instability following total knee arthroplasty. HSS journal 7, 273.

Sartori, M., Gizzi, L., Lloyd, D.G., Farina, D.J.F.i.c.n., 2013. A musculoskeletal model of human locomotion driven by a low dimensional set of impulsive excitation primitives. 7, 79.

Sharifi, M., Shirazi-Adl, A., Marouane, H.J.J.o.b., 2017. Computational stability of human knee joint at early stance in Gait: Effects of muscle coactivity and anterior cruciate ligament deficiency. 63, 110-116.

Sharifi, M., Shirazi-Adl, A., Marouane, H.J.J.o.b., 2018. Computation of the role of kinetics, kinematics, posterior tibial slope and muscle cocontraction on the stability of ACL-deficient knee joint at heel strikeTowards identification of copers from non-copers. 77, 171-182.

Sharkey, P.F., Lichstein, P.M., Shen, C., Tokarski, A.T., Parvizi, J., 2014. Why are total knee arthroplasties failing today-has anything changed after 10 years? The Journal of arthroplasty 29, 1774-1778.

Silva, M., Shepherd, E.F., Jackson, W.O., Pratt, J.A., McClung, C.D., Schmalzried, T.P., 2003. Knee strength after total knee arthroplasty<sup $>1</$ sup $>$. The Journal of Arthroplasty 18, 605-611.

Soeno, T., Mochizuki, T., Tanifuji, O., Koga, H., Murayama, T., Hijikata, H., Takahashi, Y., Endo, N., 2018. No differences in objective dynamic instability during acceleration of the knee with or without subjective instability post-total knee arthroplasty. PloS one 13, e0194221.

Song, S.J., Detch, R.C., Maloney, W.J., Goodman, S.B., Huddleston, J.I., 2014. Causes of instability after total knee arthroplasty. The Journal of arthroplasty 29, 360-364.

Stoddard, J.E., Deehan, D.J., Bull, A.M., McCaskie, A.W., Amis, A.A., 2013. The kinematics and stability of single - radius versus multi - radius femoral components related to Mid - range instability after TKA. Journal of Orthopaedic Research 31, 53-58.

Thompson, J.A., Chaudhari, A.M., Schmitt, L.C., Best, T.M., Siston, R.A., 2013. Gluteus maximus and soleus compensate for simulated quadriceps atrophy and activation failure during walking. Journal of biomechanics 46, 2165-2172.

van der Krogt, M.M., Delp, S.L., Schwartz, M.H., 2012. How robust is human gait to muscle weakness? Gait \& posture 36, 113-119.

Vera-Garcia, F.J., Elvira, J.L., Brown, S.H., McGill, S.M., 2007. Effects of abdominal stabilization maneuvers on the control of spine motion and stability against sudden trunk perturbations. Journal of Electromyography and Kinesiology 17, 556-567.

Wautier, D., Thienpont, E., 2017. Changes in anteroposterior stability and proprioception after different types of knee arthroplasty. Knee Surgery, Sports Traumatology, Arthroscopy 25, 1792-1800.

Worsley, P., Stokes, M., Taylor, M.J.G., Posture, 2011. Predicted knee kinematics and kinetics during functional activities using motion capture and musculoskeletal modelling in healthy older people. 33, 268273.

Zaffagnini, S., Bignozzi, S., Saffarini, M., Colle, F., Sharma, B., Kinov, P.S., Marcacci, M., Dejour, D., 2014. Comparison of stability and kinematics of the natural knee versus a PS TKA with a 'third condyle'. Knee Surgery, Sports Traumatology, Arthroscopy 22, 1778-1785. 
Table 1 Simulation parameters for BSL and WEAK models

\begin{tabular}{|c|c|c|c|}
\hline & & Baseline models & Weak models \\
\hline \multirow{8}{*}{$\begin{array}{l}\text { Muscle Strength (F0) } \\
(\mathrm{N})\end{array}$} & Semimembranosus & 2674-2954 & $1800-2530$ \\
\hline & Semitendinosus & 2674-2954 & $1800-2530$ \\
\hline & Biceps femoris & $2674-2954$ & $1800-2530$ \\
\hline & Rectus femoris & $1260-1386$ & $900-1188$ \\
\hline & Vastus & $6522-7200$ & $4500-6170$ \\
\hline & Tibialis anterior & $952-1053$ & $650-740$ \\
\hline & Medial gastrocnemius & $1568-1733$ & $1000-1200$ \\
\hline & Soleus & $2865-3166$ & $2000-2216$ \\
\hline \multirow{10}{*}{$\begin{array}{l}\text { Ligament force } \\
\text { parameter }(\mathrm{S} 0) \\
\qquad(\mathrm{N})\end{array}$} & PCL_a & $8550-9450$ & $5900-8100$ \\
\hline & PCL_p & $8550-9450$ & $5900-8100$ \\
\hline & MCL_a & $2613-2888$ & $1800-2475$ \\
\hline & MCL_p & $2613-2888$ & $1800-2475$ \\
\hline & MCL_i & $2613-2888$ & $1800-2475$ \\
\hline & LCL_a & $1900-2100$ & $1300-1800$ \\
\hline & LCL_p & $1900-2100$ & $1300-1800$ \\
\hline & LCL_s & $1900-2100$ & $1300-1800$ \\
\hline & MPFL & $1900-2100$ & $1300-1800$ \\
\hline & LPFL & $1900-2100$ & $1300-1800$ \\
\hline
\end{tabular}

Table 2 Anterior-posterior (A-P) displacement and internal-external(I-E) rotation for BSL and WEAK models (calculated) using FDK analysis (mean \pm std). Two different cost-functions were utilized to explore whether altered muscle recruitment strategy can mitigate the influence of defected stabilizers on knee secondary kinematics.

\begin{tabular}{|c|c|c|c|c|c|c|c|c|}
\hline \multirow[t]{2}{*}{ Variable } & \multicolumn{2}{|c|}{ Baseline models } & \multicolumn{2}{|c|}{ Models with weak muscles } & \multicolumn{2}{|c|}{ Models with lax ligaments } & \multicolumn{2}{|c|}{$\begin{array}{c}\text { Models with weak muscles } \\
\text { and } \\
\text { lax ligaments } \\
\end{array}$} \\
\hline & Cost f1* & Cost f $2 * *$ & Cost f1 & Cost f1 & Cost f1 & Cost f2 & Cost f1 & Cost f2 \\
\hline A-P range $(\mathrm{mm})$ & $5.5 \pm 4.1$ & $4.8 \pm 3.2$ & $8.3 \pm 5.8$ & $6.5 \pm 4.8$ & $6.7 \pm 5.7$ & $5.0 \pm 4.1$ & $9.7 \pm 5.6$ & $8.7 \pm 5.3$ \\
\hline I-E range (deg) & $11.6 \pm 5.7$ & $11.2 \pm 4.5$ & $15.7 \pm 8.4$ & $12.9 \pm 5.5$ & $15.3 \pm 5.4$ & $12.5 \pm 8.2$ & $18.4 \pm 8.5$ & $13.8 \pm 8.4$ \\
\hline
\end{tabular}

*Costf1: Min-Max optimization

**Costf2: Synergy optimization 


\section{Appendix}

Two deformable contact models were defined between the tibial insert and femoral component bearing surfaces and between the patellar button and the femoral component. The tibial insert was divided into medial and lateral compartments with separate contacts created for each. The contact force between the two objects, represented with the contacting surfaces (in STereoLithography (STL) format), was calculated using a linear force-penetration volume law.29 The contact pressure module PressureModule in Newton per meter cube is the key parameter in the default FDK computational framework of AnyBody. Due to the contact model implemented in AnyBody being very close to the elastic foundation theory,20 the equations derived by Fregly et al. (2003) according to the elastic foundation theory, were used for the calculation of the PressureModule :

$$
\frac{p}{d}=\frac{(1-v) E(p)}{(1+v)(1-2 v) \times h}
$$

where $\mathrm{p}$ and $\mathrm{d}$ are contact pressure and surface overclosure, respectively; and $\mathrm{E}(\mathrm{p}), \mathrm{v}$, and $\mathrm{h}$ are Young's modulus, Poisson's ratio, and the local thickness of the UHMWPE tibial layer, respectively; and d is the element's spring deflection, defined as the interpenetration of the undeformed surfaces in the direction of the local surface normal. For a non-linear material, the elastic modulus was set as a function of the current level of contact pressure for each element. The following equation was taken from a non-linear power law material model:

$$
\varepsilon=\frac{1}{2} \varepsilon_{0} \frac{p}{p 0}+\frac{1}{2} \varepsilon_{0}\left(\frac{p^{n}}{p_{0}}\right)
$$

where $\varepsilon$ is the strain, $\mathrm{p}$ is the contact pressure, $\varepsilon \mathrm{o}=0: 0597, \mathrm{po}=18: 4 \mathrm{MPa}$, and $\mathrm{n}=3$ based on the experimental stress strain data for UHMWPE (Cripton 1993). To take the derivative of p over $\varepsilon$, and replace with $E(p)=d p / d \varepsilon$, the above equation is rewritten as:

$$
E(p)=\frac{1}{\left\{\frac{1}{2} \frac{\varepsilon_{0}}{p_{0}}\left[1+n\left(\frac{p}{p_{0}}\right)^{n-1}\right]\right.}
$$

Equation (3) was substituted into equation (1) to generate a single non-linear equation for $\mathrm{p}$ and $\mathrm{d}$ which was solved using a standard root-finding method. Further details for elastic foundation contact model can be found in the literature.3,20. In this study, the UHMWPE was considered as a non-linear material, and its elastic modulus was at least two orders of magnitude lower than that of the metallic femoral component. Therefore, the contact pressure module Pressure Module was calculated from equations (1) to (3) as a function of the contact pressure $\mathrm{p}$ :

$$
\text { Pressure Module }=\frac{p A}{d A}=\frac{(1-v)}{(1+v)(1-2 v) h} \times \frac{2 p 0}{\varepsilon 0\left[1+n\left(\frac{p}{p 0}\right)^{n-1}\right]}
$$

where A is the unit contact area. Due to the range of the contact pressure over the articulating surface of UHMWPE tibial inserts from 5 to $25 \mathrm{MPa}$ during a gait cycle,31-33 the maximum, minimum, and average PressureModule values corresponding to the contact pressure values were calculated as $2.59 \mathrm{e} 11 \mathrm{~N} / \mathrm{m} 3,0.48 \mathrm{e} 11 \mathrm{~N} / \mathrm{m} 3$, and $1.24 \mathrm{e} 11 \mathrm{~N} / \mathrm{m} 3$ respectively. Similar values for the PF joint were also adopted. The effect of using different PressureModule values on the model prediction was investigated in our previous publication (Chen et al, 2014).

\section{$\underline{\text { References }}$}

Chen, Zhenxian, et al. "Prediction of in vivo joint mechanics of an artificial knee implant using rigid multi-body dynamics with elastic contacts." Proceedings of the Institution of Mechanical Engineers, Part H: Journal of Engineering in Medicine 228.6 (2014): $564-575$.

Fregly BJ, Bei YH and Sylvester ME. Experimental evaluation of an elastic foundation model to predict contact pressures in knee replacements. $J$ Biomech 2003; 36: 1659-1668

Cripton PA. Compressive characterization of ultra high molecular weight polyethylene with applications to contact stress analysis of total knee replacements. MSc Thesis, Queen's University, Kingston, ON, Canada, 1993 

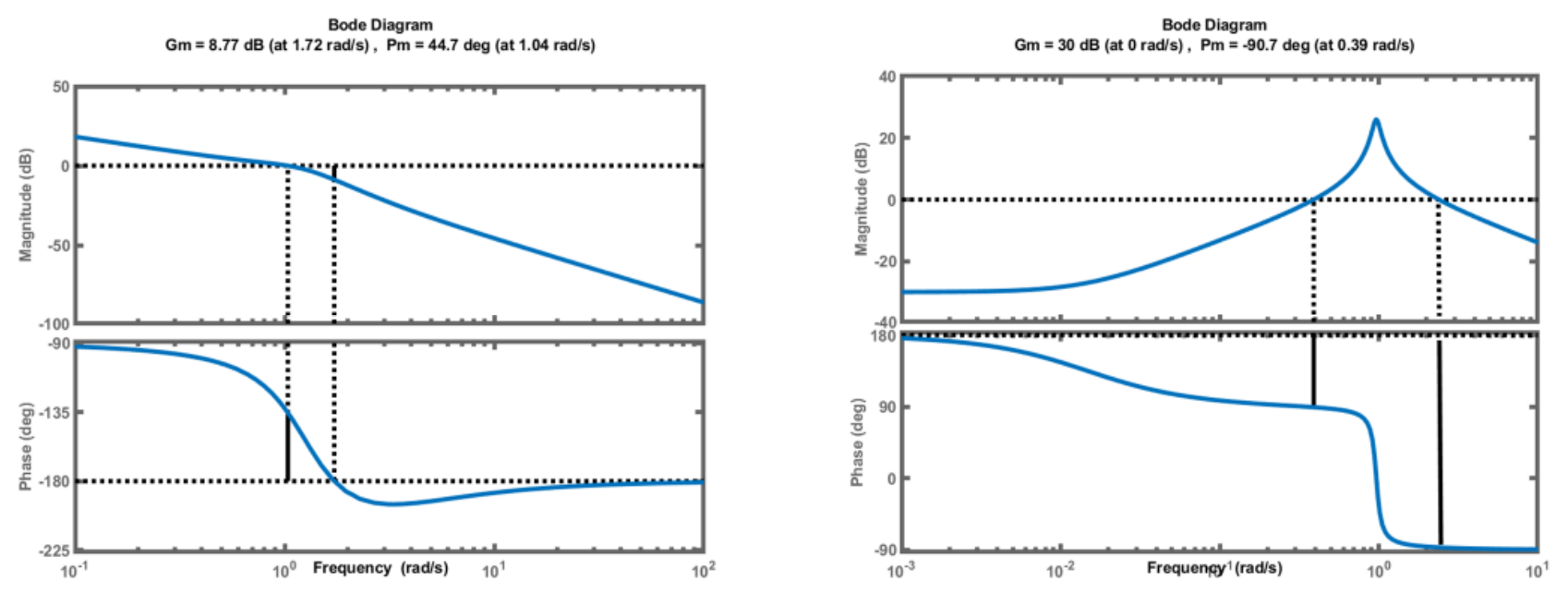

Figure A.1. Typical examples of Bode plots with positive (a) and negative (b) phase margins 


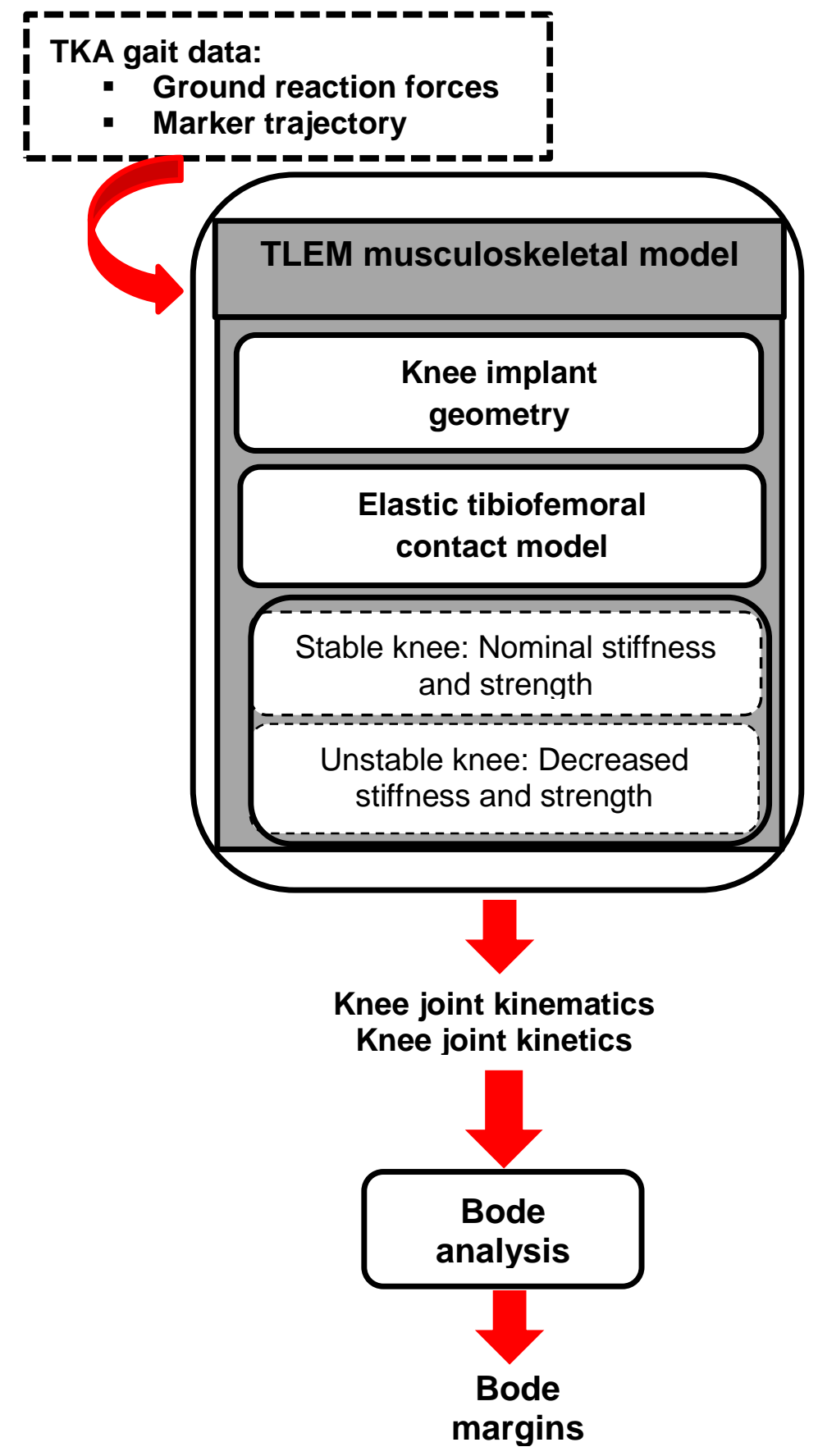

Figure 1. A schematic diagram of the modeling process used in the present study 


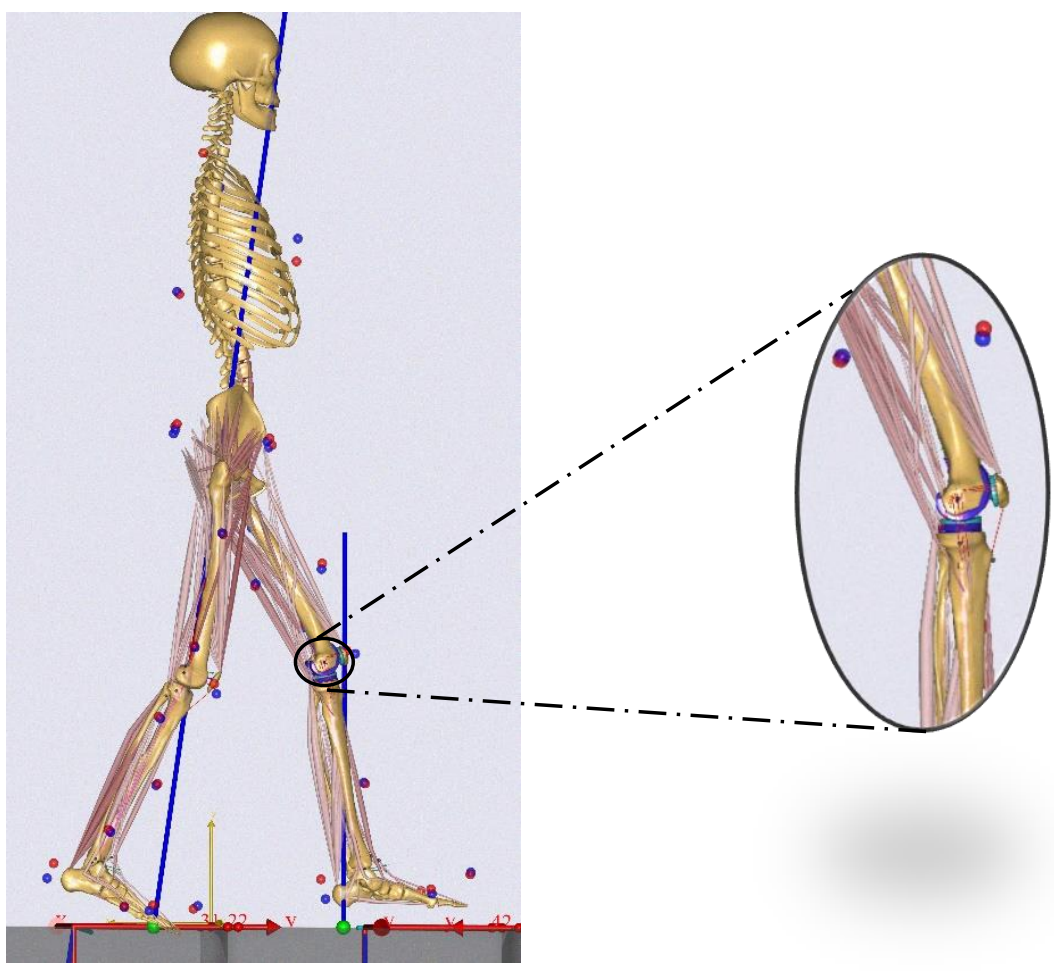

Figure 2. The TLEM MSK model was modified in AnyBody software. The geometry of knee joint was replaced with patient's implant (cruciate retaining knee implant). 


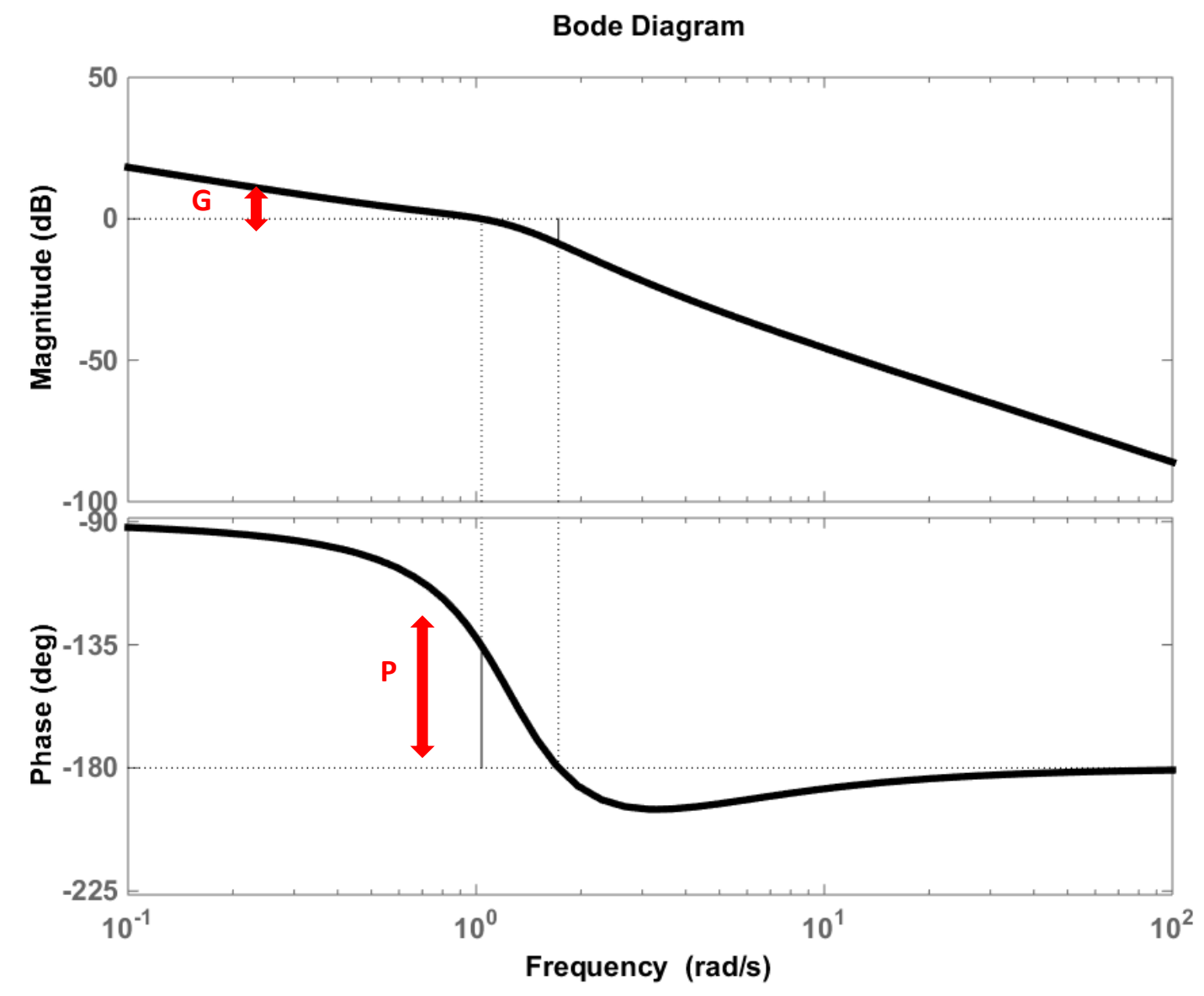

Figure 3. A typical Bode diagram to demonstrate amplitude response $(\mathrm{G})$ and phase response $(\mathrm{P})$ for a range of various frequencies (i.e., perturbation). The $\mathrm{x}$ axis demonstrates the perturbation, as the frequency of a sudden change in the inputs of knee joint model (i.e., motion and moment). The y axis in amplitude response presents the relative amplitude of knee joint power to the amplitude of perturbation. The y axis in phase response presents the time delay between when the perturbation occurs and when the peak of knee joint power is generated in response to that perturbation. Time delay is expressed in degree as gait is a periodic task and $2 л$ radian $(=360 \mathrm{deg})$ is considered as one complete cycle delay. 

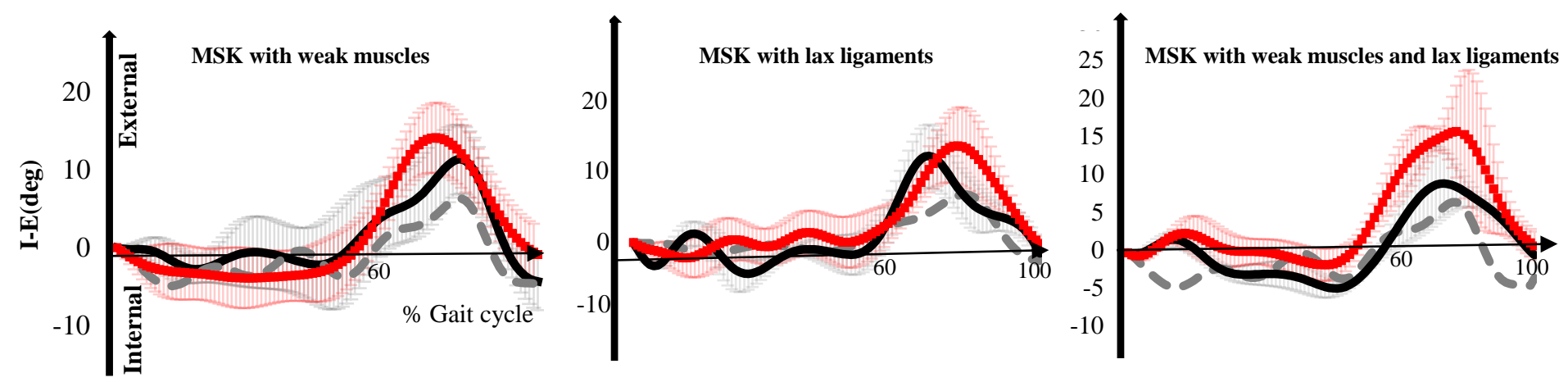

(a)
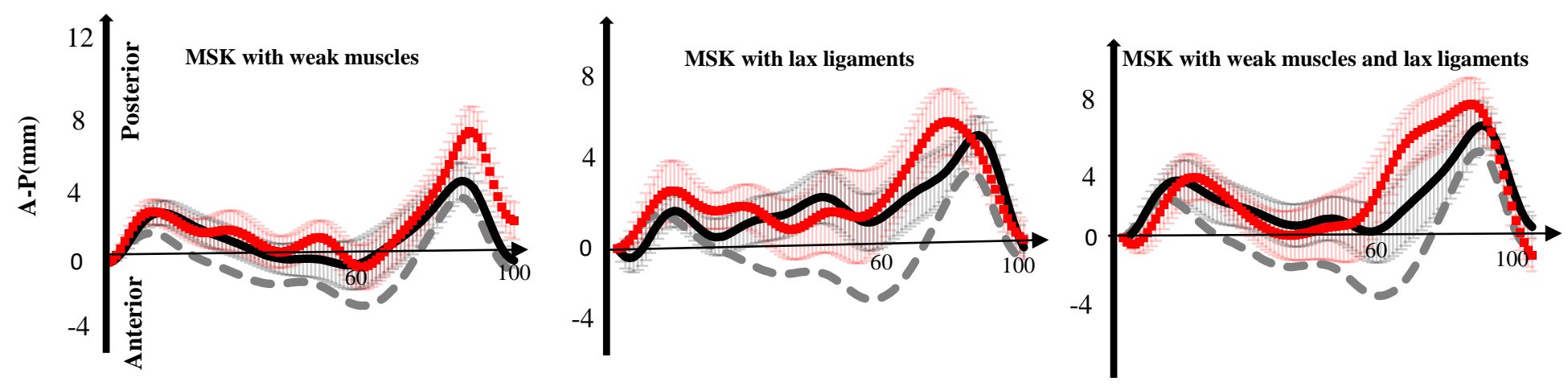

(b)

Figure 4 Internal-external (I-E) rotation (a) and anterior-posterior (A-P) displacement (b) for MSK models with weak muscles, lax ligaments and the combination of both weak muscles and lax ligaments. I-E and A-P kinematics were calculated using FDK analysis and based on two different muscle recruitment patterns: Min_Max optimization and synergy optimization. Graphs present the average and standard deviations for one representative subject. 

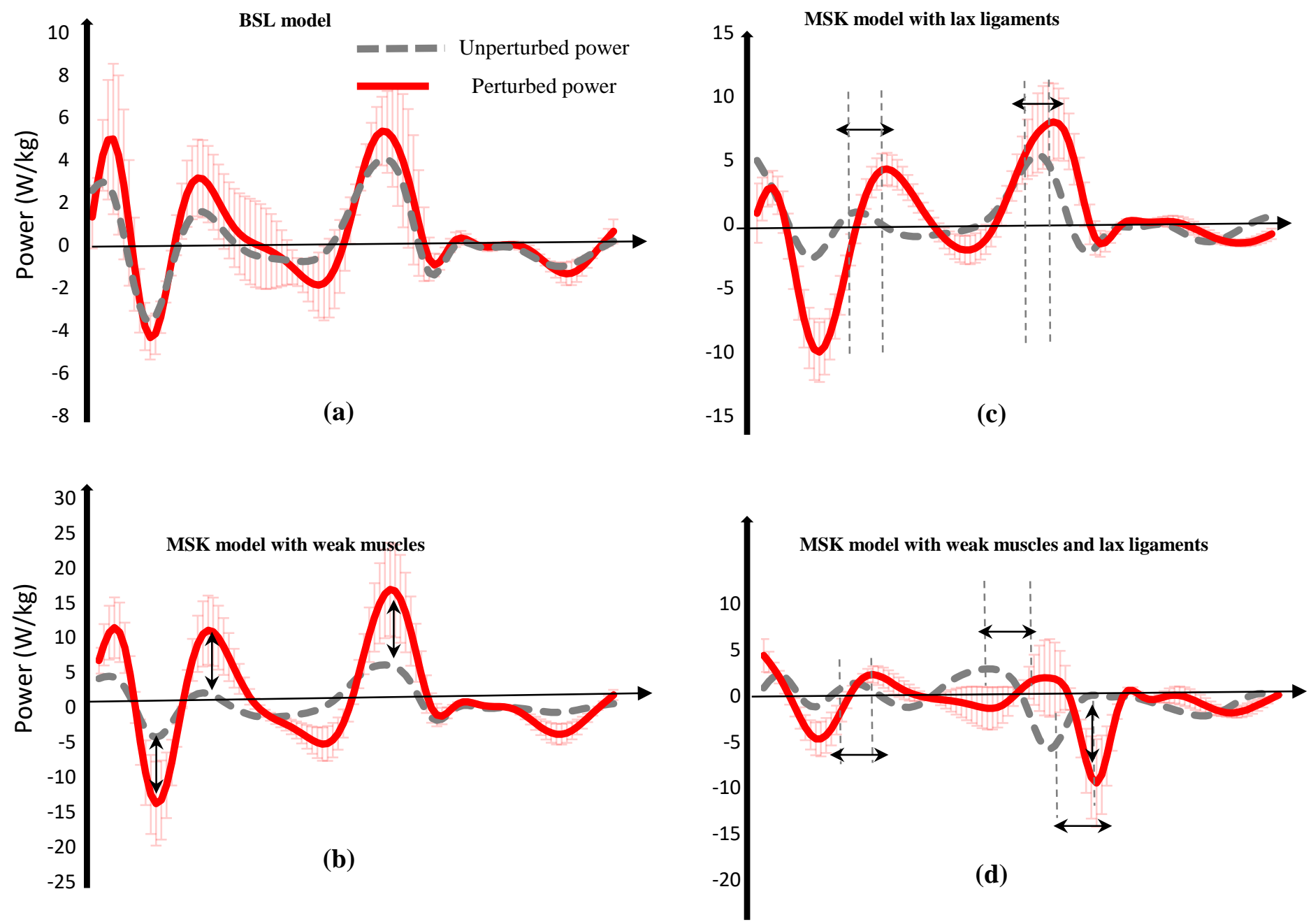

Figure 5. Comparison of perturbed knee power (calculated from Bode analysis) vs. unperturbed knee power (calculated from inverse-dynamic analysis of level-walking) for BSL models (a), models with weak muscles (b), lax ligaments (c) and models with combined deficits(d). Models with lax ligaments showed a delayed response to perturbation whilst models with weak muscles unbounded power. 